University of Wollongong

Research Online

Faculty of Engineering and Information

Faculty of Engineering and Information

Sciences - Papers: Part B

Sciences

2011

Parametric Investigation of Near End and Far End Crosstalks in Printed Circuit Board Lands

Md Masud Rana

Rajshahi University of Engineering And Technology

Md Rabiul Islam

Rajshahi University of Engineering And Technology, mrislam@uow.edu.au

Md Kamal Hosain

Deakin University

Follow this and additional works at: https://ro.uow.edu.au/eispapers1

Part of the Engineering Commons, and the Science and Technology Studies Commons

Research Online is the open access institutional repository for the University of Wollongong. For further information contact the UOW Library: research-pubs@uow.edu.au 


\title{
Parametric Investigation of Near End and Far End Crosstalks in Printed Circuit Board Lands
}

\begin{abstract}
Multi-conductor transmission line and interconnect carry signals with wide rage of frequencies from sending end to receiving end. The signal in one transmission line may be interupted by the unwanted contributions from the neighboring line conductors. If data speed increases, high frequency effects occur and the signals suffer from difficulties such as ringing, crosstalk, reflections, and ground bounce that seriously hamper the quality of the received signal. In order to estimate the signal quality, signal integrity analysis is needed. In this paper, an attempt has been made to investigate the sensitivity of the near and far end crosstalk on the parameters such as physical geometry of the conductors, electrical property of the substrate and the rise and fall time of excitation signal. The method of moments (MOM) is used to calculate the line parameters for different geometries. The simulation studies are carried out in TNT. The time domain and frequency domain analyses are performed using transmission line model of PSPICE. Moreover, a model is developed and tested in the laboratory. It is observed that the coupling inductance and capacitance vary with the variation of physical geometry and the substrate parameter.
\end{abstract}

\section{Keywords}

far, board, crosstalks, near, printed, lands, circuit, investigation, end, parametric

\section{Disciplines}

Engineering | Science and Technology Studies

\section{Publication Details}

M. Rana, M. Islam \& M. Hosain, "Parametric Investigation of Near End and Far End Crosstalks in Printed Circuit Board Lands," International Journal of Electrical and Computer Engineering, vol. 1, (2) pp. 213-219, 2011. 


\title{
Parametric Investigation of Near End and Far End Crosstalks in Printed Circuit Board Lands
}

\author{
Md. Masud Rana ${ }^{1}$, Md. Rabiul Islam ${ }^{1}$, Md. Kamal Hosain ${ }^{2}$ \\ Dept. of Electrical and Electronic Engineering, Rajshahi University of Engineering \& Technology, Rajshahi-6204, Bangladesh ${ }^{1}$ \\ School of Engineering, Deakin University, Victoria-3217, Australia ${ }^{2}$ \\ e-mail: masud_01119e@yahoo.com,rabiulbd@hotmail.com, mhosain@deakin.edu.au
}

\begin{abstract}
Multi-conductor transmission line and interconnect carry signals with wide rage of frequencies from sending end to receiving end. The signal in one transmission line may be interupted by the unwanted contributions from the neighboring line conductors. If data speed increases, high frequency effects occur and the signals suffer from difficulties such as ringing, crosstalk, reflections, and ground bounce that seriously hamper the quality of the received signal. In order to estimate the signal quality, signal integrity analysis is needed. In this paper, an attempt has been made to investigate the sensitivity of the near and far end crosstalk on the parameters such as physical geometry of the conductors, electrical property of the substrate and the rise and fall time of excitation signal. The method of moments (MOM) is used to calculate the line parameters for different geometries. The simulation studies are carried out in TNT. The time domain and frequency domain analyses are performed using transmission line model of PSPICE. Moreover, a model is developed and tested in the laboratory. It is observed that the coupling inductance and capacitance vary with the variation of physical geometry and the substrate parameter.
\end{abstract}

Keywords: Multi-conductor transmission line (MTL), method of moments, crosstalk, near end and far end

\section{Introduction}

In a multi-conductor transmission line (MTL) system, the coupling capacitance, inductance among line conductors cause crosstalk. For example in a three line system, the voltage on line 1 will contain the unwanted contributions from line 2 and 3 adjacent to it. The voltage on line 1 in a three conductor system can be expressed as (1).

$$
v_{1}=L_{11} \frac{d i_{1}}{d t}+L_{12} \frac{d i_{2}}{d t}+L_{13} \frac{d i_{3}}{d t}
$$

where $L_{11}$ is the self inductance of line $1, L_{12}$ and $L_{13}$ are the coupling inductances on line 1 due to line 2 and 3 respectively. A similar equation for the current can be obtained using self and mutual capacitances. Crosstalk contaminates desired signal and imposes a limitig factor for high speed communication. The crosstalk degrades the signal speed and integrity significantly [1]-[12]. The transmission line which carries the crosstalk is called a victim line. The aggressor line is terminated to prevent reflections. The victim line is terminated at both ends with no other loads. The cross talk will propagate in both forward and backward directions. The voltage response measured on the victim line near the original source is called near end crosstalk. The voltage response measured at the other end is called far end crosstalk. Each type has very different characteristics [1], [2]. Multi-conductor signal lines are using in many high speed analog and digital electronic printed circuit boards (PCBs), and multi-conductor telephone cables. In addition, twisted pair cables have distributed self and mutual series inductance and parallel capacitances. The transmission line which has the source signal propagated is called the aggressor line [13-21].. In this paper, a parameter dependence of the near and far end crosstalk ( NEXT and FEXT) among the PCB lands has been investigated both in time and frequency domains. Fig. 1. depicts the necessary steps which are followed in this analysis. In order to validate the calculated results experimental investigations are underway using a time domain reflectometer (TDR).

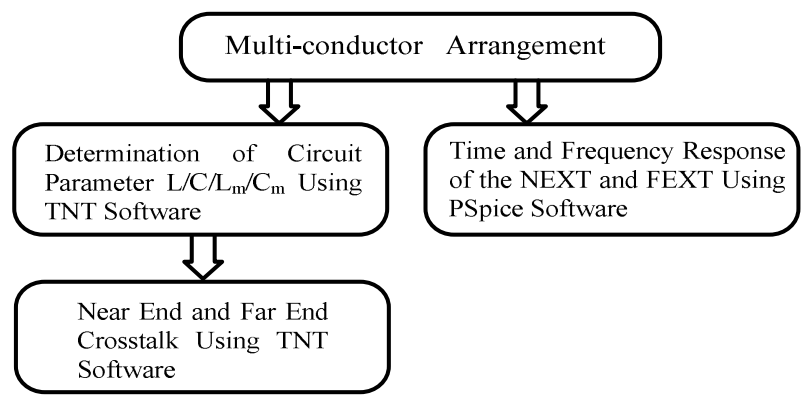

Figure 1. Block diagram for NEXT and FEXT investigation process 


\section{MTL Equations}

The multiconductor transmission line equation can be derived from the distributed parameters model [3], [5]. The distributed parameter model of an MTL system is shown in Fig. 2. The coupled differential equations can easily be written using Kirchhoff's voltage and current laws as (2) and (3).

$$
\begin{array}{r}
\frac{\partial}{\partial z} V(z, t)=-L \frac{\partial}{\partial t} I(z, t) \\
\frac{\partial}{\partial z} I(z, t)=-C \frac{\partial}{\partial t} V(z, t)
\end{array}
$$

where the entries in the voltage and current vectors $V(z, t)$ and $I(z, t)$ are given as

$$
\begin{aligned}
& V(z, t)=\left[\begin{array}{l}
V_{G}(z, t) \\
V_{R}(z, t)
\end{array}\right] \\
& I(z, t)=\left[\begin{array}{l}
I_{G}(z, t) \\
I_{R}(z, t)
\end{array}\right] \\
& L=\left[\begin{array}{l}
l_{G} l_{m} \\
l_{m} l_{R}
\end{array}\right] \\
& C=\left[\begin{array}{cc}
C_{G}+C_{m} & -C m \\
-C m & C_{R}+C_{m}
\end{array}\right]
\end{aligned}
$$

where $l_{G}, l_{R}$ and $C_{G}, C_{R}$ are per unit length self inductance and self capacitance of aggressor and victim lines respectively and, $l_{m}, C_{m}$ are mutual inductance and mutual capacitance between them that mainly responsible for crosstalk hence signal integrity [18]. Analytical and numerical methods are generally used to solve these equations for predicting the crosstalk. Determination of the line parameters as shown in (6) and (7) is the key step for solving the coupled differential equations both in time and frequency domains. Time domain numerical techniques such as finite difference time domain (FDTD) method is one of the widely used method though it has stability problem and huge computational time. The circuit model of the transmission can be designed in the SPICE/PSPICE using calculated parameters. This model can also be used to determine the time and frequency domain response of the NEXT and FEXT in the victim line [3], [5]. In this paper, the line parameters are calculated using a non commercially available numerical MTL program TNT [9]. The results are used to draw the SPICE/PSPICE model or OrCAD 10 model. PSPICE is used to simulate the time and frequency domain response for different parameters variation.

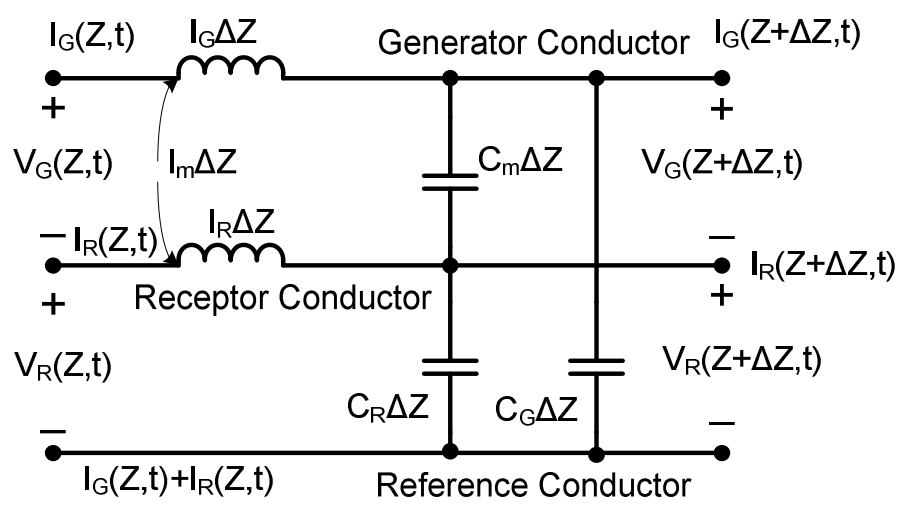

Figure 2. The per unit length distributed parameter model of a three conductors transmission line [1]

\section{Determination of Per Unit Length Parameters}

The basic structure of a three conductor PCB system is depicted in Fig. 3. The geometry of the structure is drawn in the graphical window of TNT program, and the size and parameter of the substrate is specified. The value of the per unit length parameters are calculated from the designed structure using TNT program. Along with 
parameters it can calculate the near and far end crosstalk in dB. Trapezoidal pulse is used as the excitation signal in aggressor line for matched resistive termination [13].

The leftmost line of Fig. 3. is designated as the reference conductors. The typical values of dimensions used in this calculation are $w=15$ mils, $s=47$ mils, and $h=47$ mils. The dielectric constant of $\varepsilon_{r}=4.7$ (glass epoxy) is utilized and each land are divided into 30 subsections. The computed per unit length inductance and capacitance are shown in Table 1(a) and Tabe 1(b). The per unit length capacitance vary with $\varepsilon_{r}$ but the per unit length inductance remain invariant.

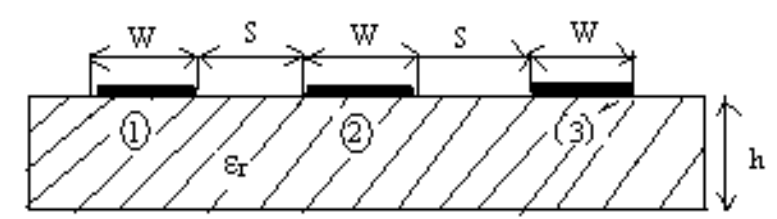

Figure 3. The layout of three lines on a PCB used for calculating per unit length capacitances

Table 1. MTL line parameters for different values of $\varepsilon_{\mathrm{r}}$

(a) Capacitance per unit length

\begin{tabular}{cccc}
\hline$\varepsilon_{r}$ & $\begin{array}{c}C_{11} \\
(\mathrm{pF} / \mathrm{m})\end{array}$ & $\begin{array}{c}C_{12} / C_{21} \\
(\mathrm{pF} / \mathrm{m})\end{array}$ & $\begin{array}{c}C_{22} \\
(\mathrm{pF} / \mathrm{m})\end{array}$ \\
\hline 2.94 & 39.694150 & -8.8280570 & 39.707182 \\
3.02 & 40.507230 & -8.9294670 & 40.520639 \\
3.90 & 49.432774 & -10.450446 & 49.450553 \\
4.26 & 53.076817 & -10.454460 & 53.096310 \\
4.70 & 57.526567 & -11.806769 & 57.547498 \\
4.80 & 8.537439 & -11.881779 & 58.559116 \\
6.15 & 72.167994 & -14.270159 & 72.195527 \\
\hline
\end{tabular}

(b) Inductance per unit length

\begin{tabular}{cccc}
\hline$\varepsilon_{r}$ & $\begin{array}{c}L_{11} \\
(\mu \mathrm{H} / \mathrm{m})\end{array}$ & $\begin{array}{c}L_{12} / L_{21} \\
(\mu \mathrm{H} / \mathrm{m})\end{array}$ & $\begin{array}{c}L_{22} \\
(\mu \mathrm{H} / \mathrm{m})\end{array}$ \\
\hline 2.94 & 0.61066953 & 0.1705723 & 0.61066913 \\
3.02 & 0.61066953 & 0.1705723 & 0.61066913 \\
3.0 & 0.61066953 & 0.1705723 & 0.61066913 \\
4.26 & 0.61066953 & 0.1705723 & 0.61066913 \\
4.70 & 0.61066953 & 0.1705723 & 0.61066913 \\
4.80 & 0.61066953 & 0.1705723 & 0.61066913 \\
6.15 & 0.61066953 & 0.1705723 & 0.61066913 \\
\hline
\end{tabular}

\section{Calculation of NEXT and FEXT by TNT in dB}

The relative magnitudes of the NEXT and FEXT are calculated for the aforementioned configuration (Fig. 3.) and the results are shown in Fig. 4(a), Fig. 4(b), Fig. 4(c) and Fig. 4(d). It is observed from the results that the magnitude of NEXT and FEXT dependent on $\varepsilon_{\mathrm{r}}$. An increase of $\varepsilon_{\mathrm{r}} \square$ increases the value of the coupling capacitance that is consequently boost crosstalk.

\section{Time Domain and Frequency Domain Analysis of NEXT anf FEXT}

The equations (2) and (3) are coupled equations. They could be uncoupled to modal equations using similarity transformation. This technique has been developed in [2]. The complete SPICE model for the PCB structure is shown in Fig. 5 (a) and corresponding node numbering for the SPICE subcircuit model is also shown in Fig. 5 (b). The time domain analysis of NEXT and FEXT are made considering CW sine and periodic pulse signal. Signal is applied to the near end of the multiple lines and signal integrity is monitored by mixing of signals due to crosstalk. Fig. 6 (a) and Fig.(b) depict the NEXT and FEXT, respectively, for a $10 \mathrm{~V}$ (p-p) and $0.5 \mathrm{GHz}$ sine signal applied in conductor 1 of PCB. The line parameters as shown in table 1 and 2 , and $R_{S}=R_{L}=Z_{C l}=195.6 \Omega, R_{N E I}=$ $R_{F E 1}=Z_{C 2}=147.5 \Omega, R_{N E 2}=R_{F E 2}=Z_{C 3}=94.41 \Omega, R_{N E 3}=R_{F E 3}=Z_{C 4}=204.33 \Omega$ are used in the SPICE model.

In order to investigate the signal contamination, two lines are excited by two signals, one $10 \mathrm{~V}$ (p-p), 0.5 $\mathrm{GHz}$ sine signal applied in land 1 and other $10 \mathrm{~V}, 10 \mathrm{~ns}$ time period pulse signal applied in land 2 and the near and far end signals in the lines are monitored. The results are shown in Fig. 7(a) for far end. It can easily be visualized 
from the figures that the original signal on both lines are contaminated by the crosstalk contribution from others. In order to monitor the signal integrity experimentally, a sinusoidal signal is applied between the ground and first conductor and a square wave is applied between the second conductor and ground. It has been observed that the second conductor signal is contaminated by the signal in the first conductor due to crosstalk. The oscillogram of the experiment is shown in Fig. 7 (b) and that agreed with the simulated result presented in Fig. 7 (a)

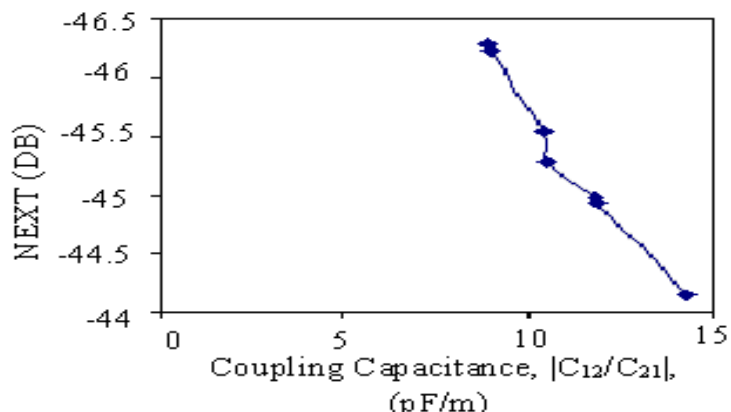

(a)

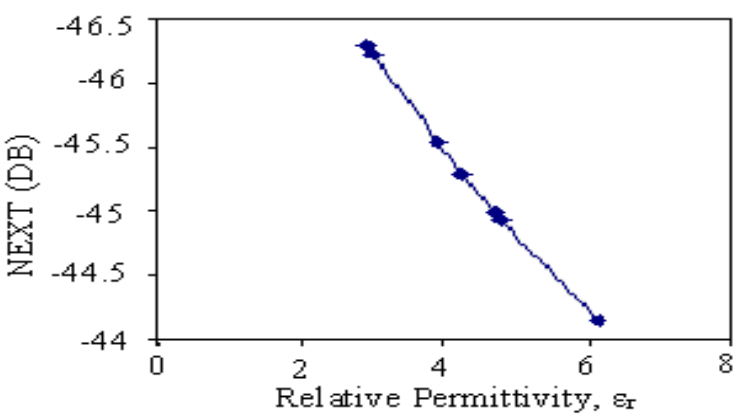

(c)

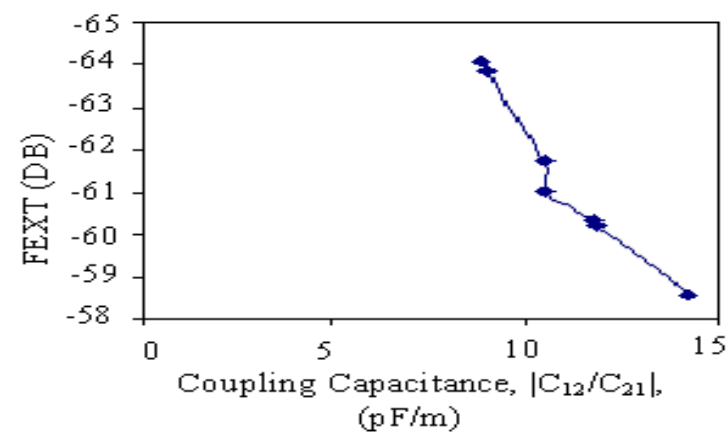

(b)

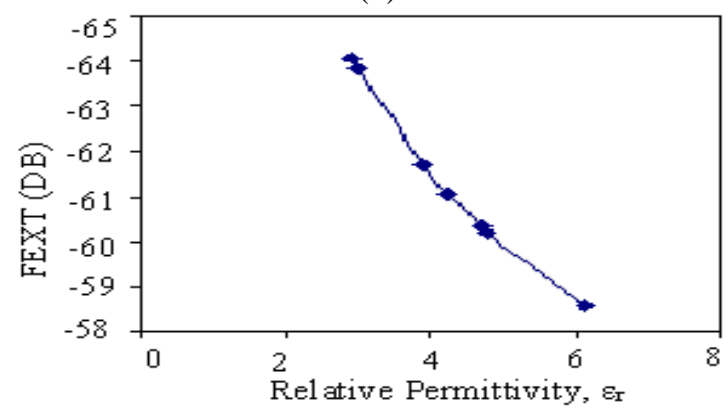

(d)

Figure 4. (a) NEXT versus coupling capacitance, (b) FEXT versus coupling capacitance, (c) NEXT versus $\varepsilon_{r}$ and (d) FEXT versus $\varepsilon_{r}$

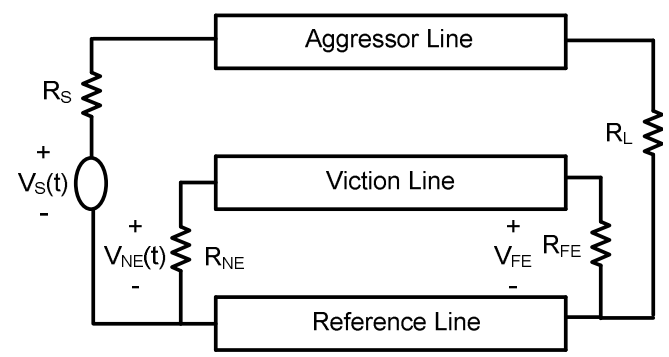

(a)

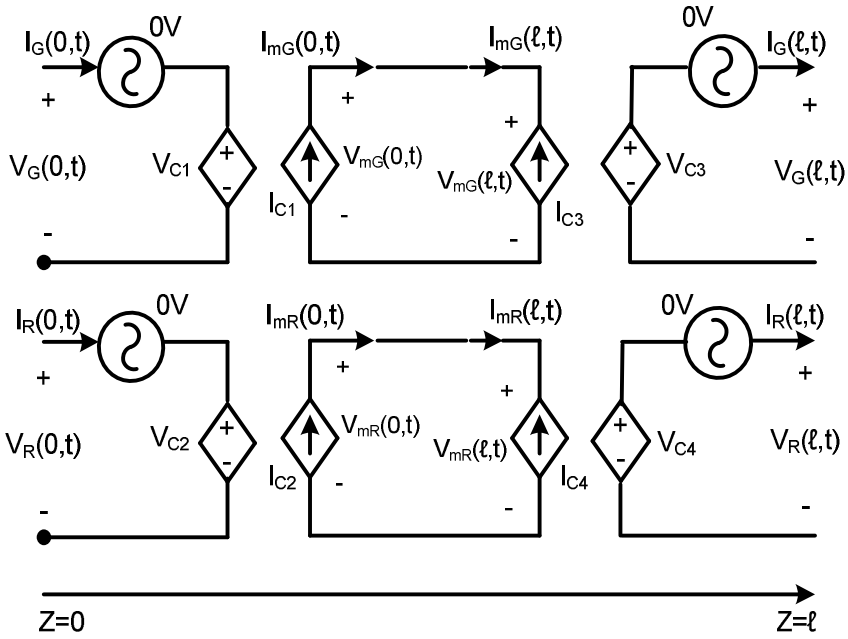

(b)

Figure 5. (a) Three conductor line model (b) The complete SPICE model [2], [8] 


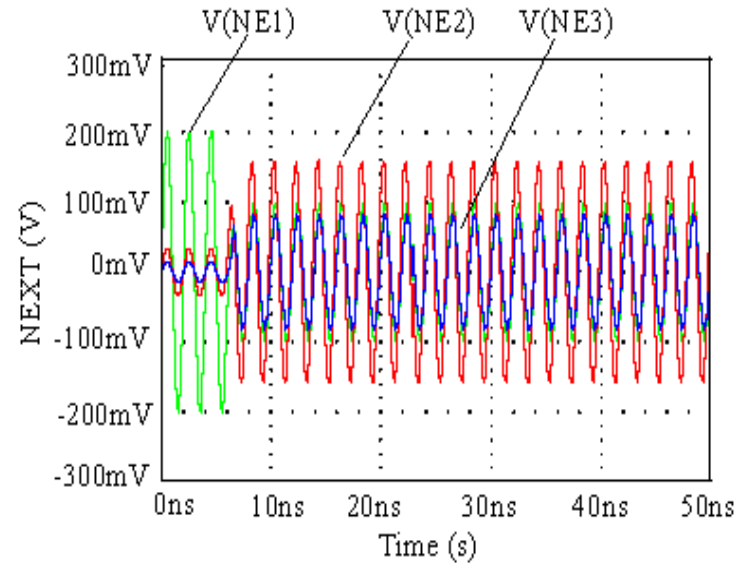

(a)

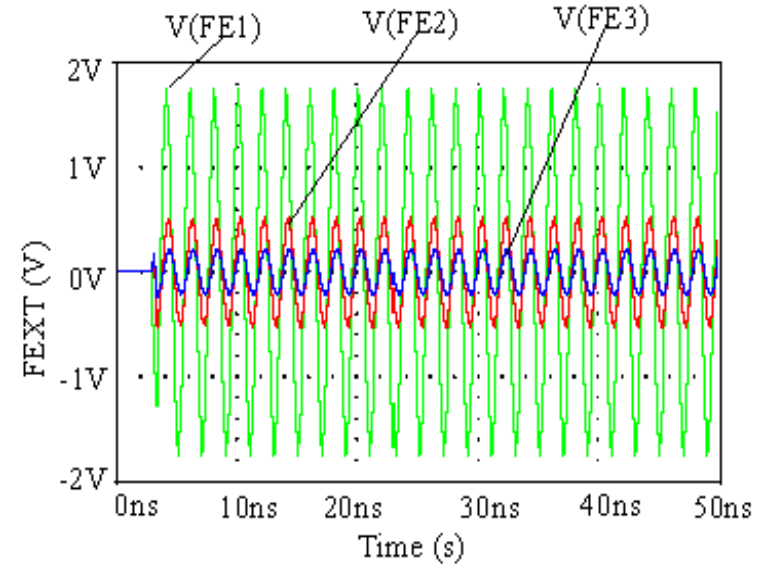

(b)

Figure. 6 (a) NEXT and (b) FEXT for sinusoidal signal in the first conductor for $\varepsilon_{r}=3.9, w=15$ mils, $d=45$ mils, $h=47$ mils.

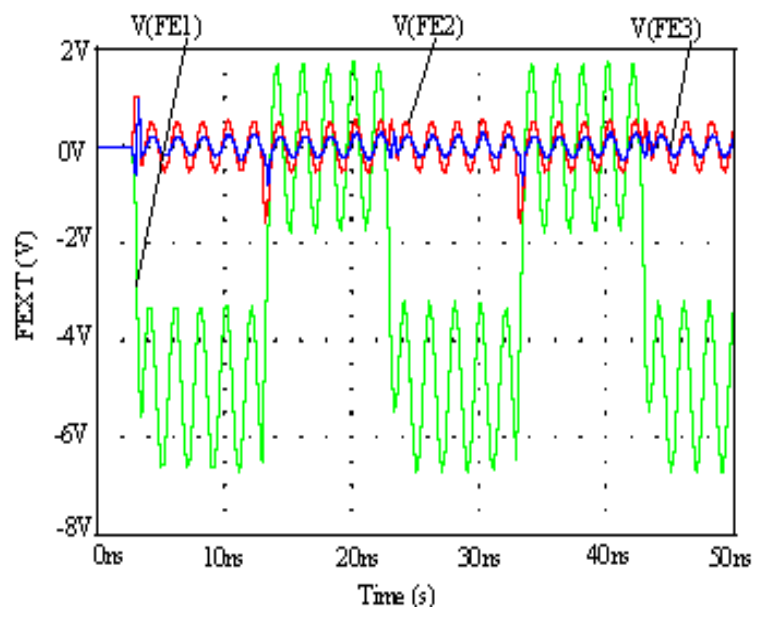

(a)

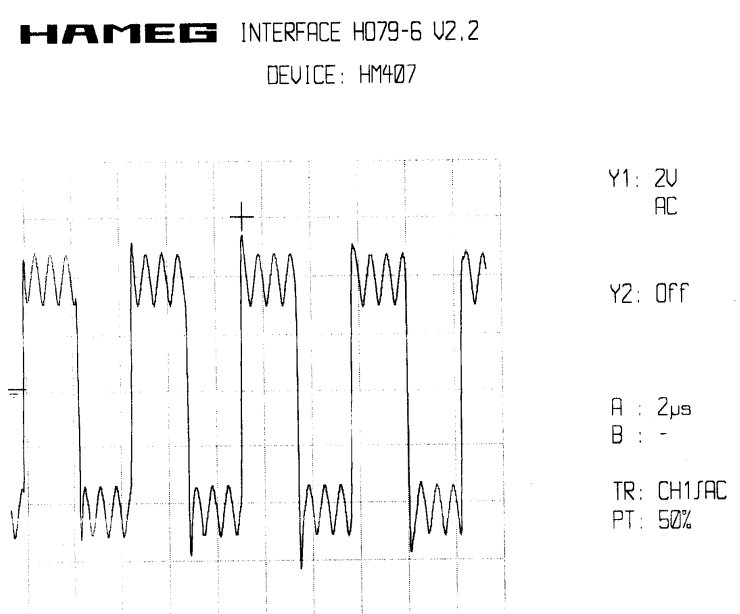

(b)

Figure 7. Signal contamination due to contributions from signals from neighboring lines. (a) Far end signals and (b) Measured signal at the far end $\left(h=62\right.$ mils, $\varepsilon_{r}=3.9, w=15$ mils, $d=45$ mils $)$.

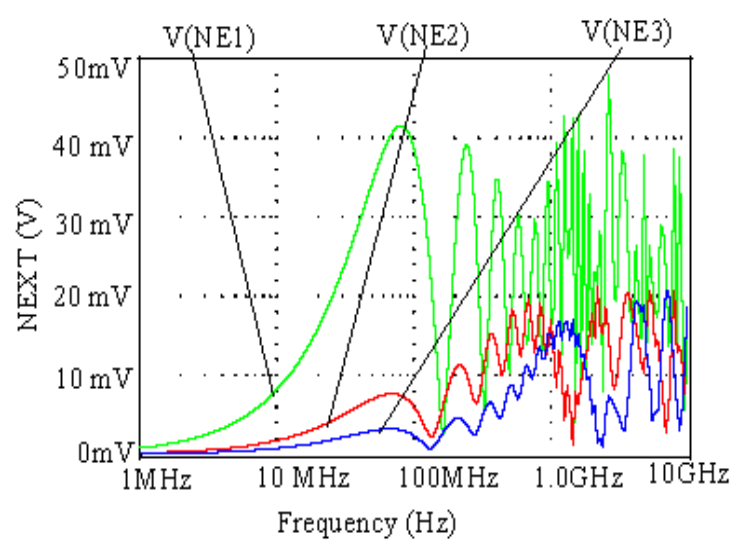

(a)

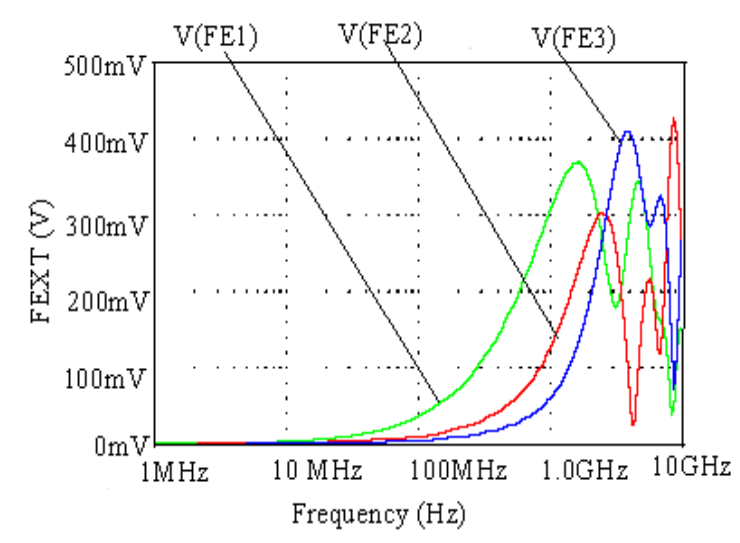

(b)

Figure 8. (a) NEXT and (b) FEXT in frequency domain. The signal is applied in the first line. 
The sine signal in the first line is coupled with the pulse train in the second line and the signal integrity is lost eventually. As the far end terminations on the lines are not perfectly matched, the far end signals are contaminated greatly due to the reflection. The frequency domain analysis is performed using sinusoidal source $1 \mathrm{~V}$ (p-p). The source frequency is sweeped from $1 \mathrm{MHz}$ to $10 \mathrm{GHz}$. The results are shown in Fig. 8 (a), (b). It can be seen from the figure that magnitude of NEXT and FEXT increases with frequency and at high frequency region some undulation appears and this will limit the speed of signal transmission over the MTL structure.

\section{Conclusion}

This paper demonstrates and measures near end and far end crosstalk and signal integrity in MTL structure etched on PCB. It has been observed that the magnitude of NEXT and FEXT are very sensitive with the size of conductors and substrate parameters. The rise and fall time of excitation signal also affect the NEXT and FEXT. For a fixed set of parameters the magnitude of the crosstalk increases with increasing frequency. This type of analysis is particularly important to design PCB layout and interconnects that handle high speed data.

\section{References}

[1] C. R. Paul, “Analysis of Multi-conductor Transmission Lines,” John Wiley \& Sons, Inc., New York, NY, 1994

[2] K. L. Kaiser, Transmission lines, matching, and crosstalk. Boca Raton, Fla.: Taylor \& Francis, 2005.

[3] C. R. Paul, Introduction to electromagnetic compatibility, 2nd ed. Hoboken, N. J. Chichester: Wiley Interscience; John Wiley [distributor], 2006.

[4] S. W. Park, et al., "Analysis of crosstalk between finite-length multiconductor transmission lines on a PCB by using circuitconcept approach," presented at IEICE Transactions, 2005, pp.3189-3194.

[5] C. R. Poul, "Solution of the transmission-line equations under the weak-coupling assumption," , IEEE Transactions on Electromagnetic Compatibility, vol. 44, no. 3, pp. 413-423. Aug 2002

[6] H. Ding, et al., "The crosstalk characteristics of different PCB layouts with multi-conductor transmission line," International Journal of Numerical Modeling: Electronic Networks, Devices and Fields, vol. 17, no. 2 , pp. 135 - 146. 2004

[7] C. R. Paul, "Modeling electromagnetic interference properties in printed circuit boards," IBM Journal of Research and Development, pp. 33-50, vol. 33, no. 11989

[8] V. K. Tripathi, et al., "A SPICE model for multiple coupled microstrips and other transmission lines," IEEE Transactions on Microwave Theory and Techniques, vol. 33, pp. 1513-1518 1985.

[9] F. Xiao, et al., "Modeling and analysis of crosstalk between differential lines in high-speed interconnects," Piers 2007 Prague: Progress in Electromagnetics Research Symposium, pp. 488-492, 2007.

[10] D. A. Hill, et al., "Crosstalk between micristrip transmission lines," IEEE Transactions on Electromagnetic Compatibility, vol. 36, no. 4, pp. 314-32, August 2002.

[11] P. Benedek, et al., "Capacitance of parallel rectangular plates separated by a dielectric sheet," IEEE Transactions on Microwave Theory and Techniques, vol. 20, no. 8, pp. 504-510, January 2003.

[12] G. Antonini, et al., "An improved method of modeling lossy transmission lines in finite-difference, time-domain analysis" IEEE International Symposium on Electromagnetic Compatibility, vol. 1, pp. 435 - 439, August 2002

[13] A. Roye al., "Simple crosstalk model of three wires to predict nearend and farend crosstalk in an emi/emc environment to facilitate emi/emc modeling," Progress In Electromagnetics Research B, vol. 8, pp. 43-58, 2008

[14] H. Chen, et al., "A synthetic design of eliminating crosstalk within MTLs," Progress in Electromagnetic Research, PIER 76, pp. 211-221, 2007.

[15] C. Zeng, et at., "Near-end crosstalk mitigation in ADSL systems," IEEE journal on selected areas in communications, vol. 20, no. 5, pp. 949 - 958, August 2002

[16] N. Holte, "An analytical model for the probability distributions of crosstalk power sum for a subset of pairs in a twisted pair cable," IEEE Globcom' 04, vol. 1, pp. 313-317, January 2005.

[17] C. C. Chai, et al., "Simple time-domain expressions for prediction for crosstalk on coupled microstrip lines," Progress in Electromagnetic Research, PIER 39, pp. 147-175, 2003.

[18] B. Z. Wang, et al., "On the generalized transmission-line theory," Journal of Electromagnetic Waves and Applications, vol. 19, no. 3, pp. 413-425, 2005.

[19] Y. S. Cheng et al., "Fewest vias design for microstrip guard trace by using overlying dielectric," Electrical Performance of Electronic Packaging, 2008 IEEE-EPEP, pp. 321 - 324, November 2008.

[20] S. Grivet-Talocia, et al., "Weak boundary treatment for high-order transient analysis of MTLs" IEEE International Symposium on Electromagnetic Compatibility, vol.1, pp. 409 - 414, August 2002.

[21] C. R. Paul, "Transmission lines in digital and analog electronic systems: signal integrity and crosstalk" IEEE Press ; Hoboken, N.J. : Wiley, 2010.

\section{Bibliography}

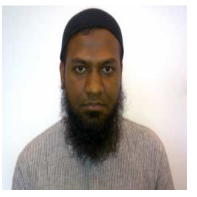

Md. Masud Rana was born in Kushtia, Bangladesh, on May 7, 1983. He received B.Sc degree from rajshahi university of engineering and technology (RUET), Rajshshi, Bangladesh in 2006. He is presently a lecturer in the department of EEE, RUET, Rajshahi, Bangladesh. His research interests include EM wave propagation modeling, analytical and computational electromagnetics, antenna theory and design, and wireless communications. 

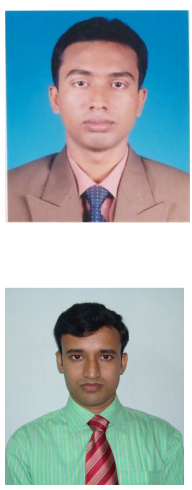

Md. Rabiul Islam was born in Rangpur, Bangladesh in 1980. He received the B.Sc. and M.Sc. degree from rajshahi university of engineering and technology (RUET), Bangladesh in 2003 and 2009 respectively, both in electrical and electronic engineering. From 2005 to 2008, he lectured in the department of electrical and electronic engineering, RUET. Since June 2008 he has been working as an Assistant Professor in the same department. His research interests are in the fields of electronic circuits, power electronic converters, renewable energy systems and smart grid.

Md. Kamal Hosain was born in Bangladesh in 1984. He achieved his B.Sc. Engineering degree from khulna university of engineering \& technology (KUET) in 2001 and he is working as a lecturer in the department of electronics and telecommunication engineering (ETE) of rajshahi university of engineering \& technology (RUET), Bangladesh. At present he is a PhD student of deakin university, Australia. His research interests include RF communication system, RF devices, antenna and its application on the biomedical engineering, brain stimulation system etc. 